\title{
Monte Carlo Simulation of Surface Semi-Spherical Inclusions Using MC X-ray
}

\author{
Samantha Rudinsky ${ }^{1}$, Nicolas Brodusch ${ }^{1}$, and Raynald Gauvin ${ }^{1}$ \\ ${ }^{1}$ Department of Mining and Materials Engineering, McGill University, Montreal, Canada
}

Microstructure characterization is an important part in the development of new materials with improved mechanical properties. In the case of metal alloys, heat treatments are used to ensure nucleation of precipitates whose size and distribution dictate the mechanical properties of the ma-terial. As an example, although the light weight of magnesium makes it an interesting candidate for automotive uses, its poor formability requires that it be alloyed with other elements to increase its strength and ductility [1, 2]. $\mathrm{Mg}-0.2 \mathrm{Al}-0.3 \mathrm{Ca}$ is a $\mathrm{Mg}$ alloy that is known for its good castability [3]. In order to ensure the desired properties are obtained, the precipitate size must remain on the order of nanometers and they must be distributed evenly throughout the $\mathrm{Mg}$ matrix. It is therefore vital that such precipitates be properly characterized in terms of composition and morphology. At such a scale, conventional transmission electron microscopy (TEM) has consistently been the instrument of choice for characterization, however the sample preparation is a difficult and lengthy process. As a result, scanning electron microscopy (SEM) has become a viable method for characterizing microstructure with nanoscale features. With constant improvements being made in the equipment, it is now possible to obtain compositional information of nano-sized precipitates in bulk samples by energy-dispersive spectroscopy (EDS) and back-scattered electron (BSE) imaging [4]. However, in order to obtain quantitative information from these processes, experimental data must be processed with the aid of Monte Carlo simulation methods. Such methods employ forward scattering random walk type calculations to predict properties such as Xray emission yield. This information can be used to adjust the experimental data obtained to account for effects such as fluorescence and absorption [5].

An important aspect that must be considered when performing Monte Carlo calculations is the geometry of the specimen. MC X-ray is a Monte Carlo software that performs 3D simulations to obtain X-ray intensity information while taking into account various specimen geometries [5]. Currently, spherical inclusions inside the specimen and prism inclusions at the surface are possible geometries that may be used [6]. Often the precipitates visible at the surface are those whose compositional information is desired. It is therefore of interest to perform simulations using geometries that can accommodate this situation. This work will incorporate a geometry consisting of a semi-spherical particle at the surface of a material into MC X-ray. Simulations of X-ray yield will be performed and compared to EDS spectra of nano-sized precipitates at the surface of an Mg-0.2Al-0.3Ca alloy.

In order to obtain experimental EDS results, the bulk alloy was prepared by polishing followed by ion milling in order to remove the surface oxide layer and image the underlying microstructure. BSE images and EDS spectra were obtained at $5 \mathrm{keV}$ using a Hitachi SU8000 FE-SEM with a $80 \mathrm{~mm}^{2} \mathrm{X}_{-\mathrm{Max}^{\mathrm{N}}}$ Silicon Drift EDS detector (Oxford Instruments, UK). An experimental BSE image of the surface is shown in Fig 1(a). An EDS spectrum of one of the circular precipitates was obtained and is shown in Fig 1(b). Preliminary Monte Carlo simulations were computed with a geometry of a rectangular prism composed of the $\mathrm{Al}_{2} \mathrm{Ca}$ phase situated at the surface of bulk Mg. Line scans were computed for 5 and 15 $\mathrm{keV}$. The resulting normalized intensities for each element are shown in Fig 2. Although the intensities are low, at $5 \mathrm{keV}$ both $\mathrm{Al}$ and $\mathrm{Ca}$ can be distinguished at identified. 
Microsc. Microanal. 24 (Suppl 1), 2018

At $15 \mathrm{keV}$, the accelerating voltage is too high and the electron depth is larger than the size of the precipitate resulting in no detection of the alloying elements. Such data can already be used for calibrations in quantitative analyses however the addition of a semi spherical geometry into the program will increase its versatility and the accuracy of such calculations.

\section{References:}

[1] M. H. Yoo. Metallurgical Transactions A 12 (1981), p. 409.

[2] J. Su et al. Materials Science and Engineering: A 587 (2013), p. 27.

[3] J. Su et al. "Precipitation Behaviour of Micro-Alloyed Mg-Al-Ca Alloys during Heat Treatment and Hot Compression". Magnesium Technology 2012. (John Wiley \& Sons, Inc.), p. 317.

[4] N. Brodusch et al. Microsc. Microanal. 18 (2012), p. 1393.

[5] R Gauvin and P Michaud. Microsc. Microanal. 15 (2009), p. 488.

[6] R. Gauvin, P. Hovington, and D. Drouin. Scanning 17 (1995), p. 202.

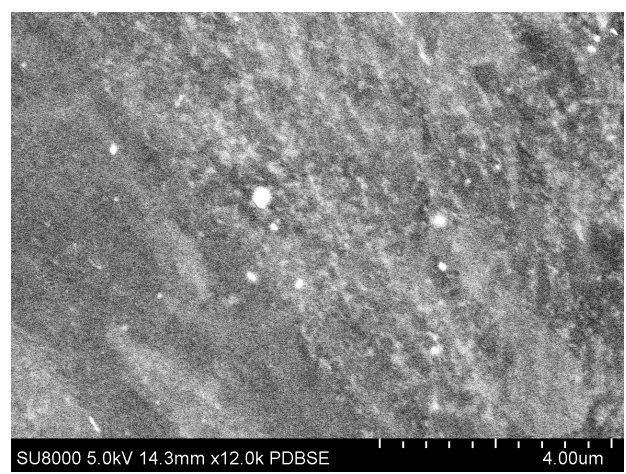

(a)

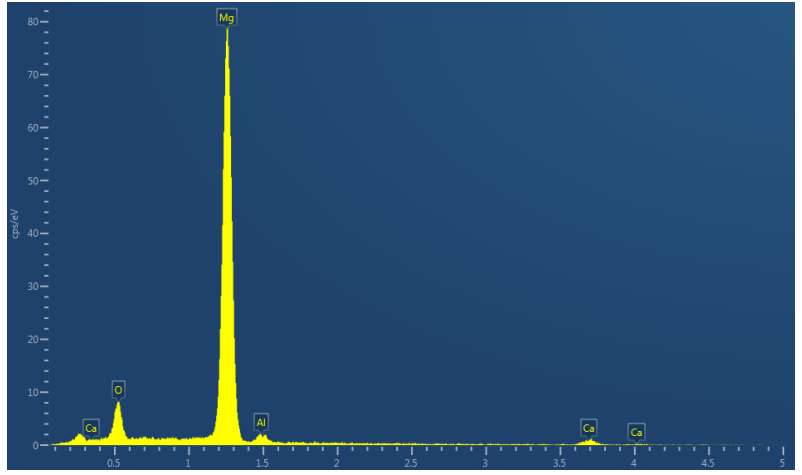

(b)

Figure 1. (a) BSE image of Mg alloy and (b) associated EDS spectrum of the circular precipitate.

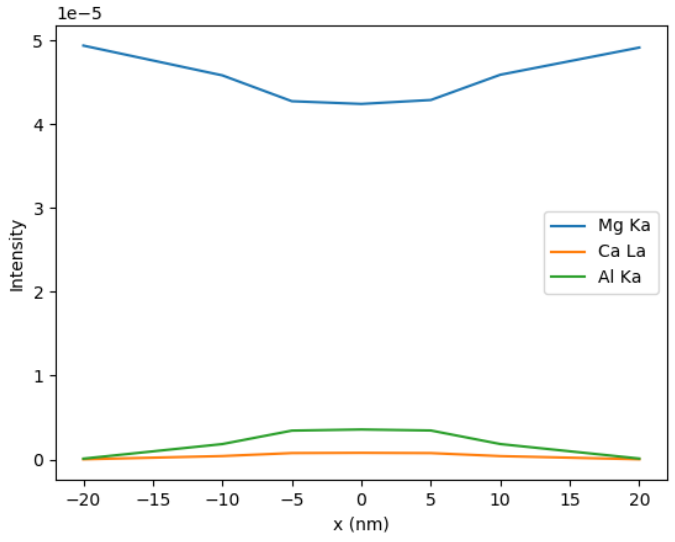

(a)

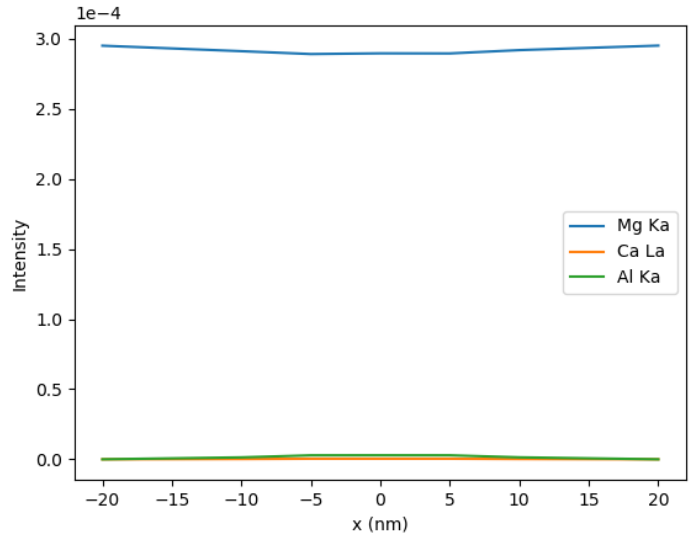

(b)

Figure 2. Monte Carlo simulation of X-ray intensity line scans at (a) $5 \mathrm{keV}$ and (b) $15 \mathrm{keV}$. 